\title{
ACTIVE REGIONS AT MILLIMETER WAVELENGTHS AND THE MEASUREMENT OF MAGNETIC FIELDS
}

\author{
M. R. KUNDU \\ Astronomy Program, University of Maryland, College Park, Md., U.S.A.
}

\begin{abstract}
Some properties of solar active regions at 9 and $3.5 \mathrm{~mm}$ wavelengths are discussed. The regions have excess brightness temperatures of up to 1000 and $700 \mathrm{~K}$ at 9 and $3.5 \mathrm{~mm}$ wavelengths respectively. The background radiation at $3.5 \mathrm{~mm}$ is often seen to be 'absorbed' in regions closely coincident with $\mathrm{H} \alpha$ dark filaments on the disk. Interpretation of this 'absorption' as due to the large optical thickness of the overlying filamentary material leads to an estimate of electron density in the filaments. The active regions at millimeter wavelengths show almost one-to-one correspondence with the Ca-plage regions as well as with the regions of longitudinal magnetic fields on Mt. Wilson magnetograms. A comparison of the mm- $\lambda$ maps with the magnetograms 'smoothed' with the beams of $\mathrm{mm}$ observations shows this correspondence in a striking manner. This relationship suggests the possibility of measuring chromospheric magnetic fields from the measurement of polarization at millimeter wavelengths.
\end{abstract}

In this paper, I shall discuss some high resolution observations of solar active regions at millimeter wavelengths and their possible usefulness in the measurement of chromospheric magnetic fields. The observations discussed here were taken at 9 and $3.5 \mathrm{~mm}$ wavelengths with the 36- $\mathrm{ft}$ radio telescope at the National Radio Astronomy Observatory, situated at Kitt Peak in Tucson, Arizona. The half-power beamwidths of the telescope are 3.5 and 1.2 arc respectively at 9 and $3.5 \mathrm{~mm}$ wavelengths.

The observing procedure has been discussed previously (Kundu, 1970); it consists of scanning the Sun in right ascension at the rate of $1^{\circ}$ per minute, the successive scans being separated by $1^{\prime}$ of arc in declination. A complete solar map consists of a $45^{\prime}$ by $37^{\prime}$ arc grid of data points, centered on the Sun. Typical maps at 9 and $3.5 \mathrm{~mm}$ wavelengths are shown in Figure 1. The active regions were scanned repeatedly over a RA range of $35^{\prime}$ arc and at declination intervals of $1^{\prime}$ arc over a suitable declination range in which one or more dominant active regions are located. These scans are repeated at time intervals of approximately $d$ minutes, where $d$ is the declination range in arc minutes. A typical set of active region maps at $3.5 \mathrm{~mm}$ successively taken over a period are shown in Figure 2.

The maps at 3.5 and $9 \mathrm{~mm}$ are quite similar, although the maps at $3.5 \mathrm{~mm}$ show considerably more structure than the $9 \mathrm{~mm}$ maps, obviously due to higher angular resolution. The maps at 9 and $3.5 \mathrm{~mm}$ both show sources whose peak excess brightness temperatures can reach up to $1000 \mathrm{~K}$ and $700 \mathrm{~K}$ respectively, representing about $10-15 \%$ of the quiet Sun radiation at these wavelengths. The regions at 9 and $3.5 \mathrm{~mm}$ are distributed practically all over the disk. Such a distribution is typical of Calcium plage regions. Indeed the 9 and $3.5 \mathrm{~mm}$ regions have very close correspondence in size and intensity with the plage regions. The intense sources at both 9 and $3.5 \mathrm{~mm}$ usually appear in pairs; these correspond to bipolar regions on magnetograms. A complex region at $3.5 \mathrm{~mm}$ consisting of three or more components usually corresponds 


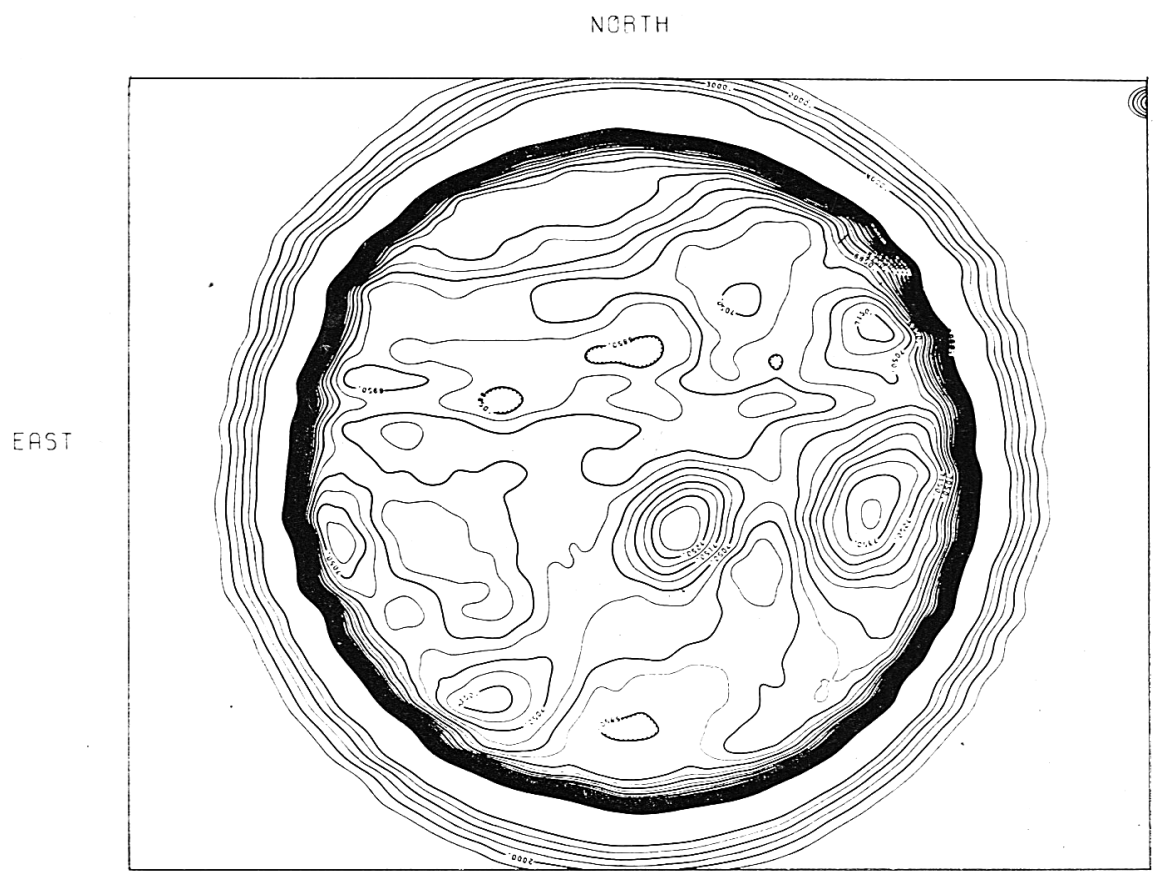

Fig. 1a. Solar Map at $9 \mathrm{~mm}-\lambda$ for Sept. 17, 1969. The grid used was $45^{\prime}$ by $37^{\prime}$ in RA and $\delta$ at intervals of $1^{\prime}$ arc in $\delta$. The regions marked by small dashes indicate temperature depressions (from Kundu, 1970).

to an equally complex region on magnetograms. Such complex structure is greatly smoothed out on the $9 \mathrm{~mm}$ maps because of lower resolution, although the existence of several components can often be discerned. Figure 3 shows the magnetogram which should be compared with the 3.5 and $9 \mathrm{~mm}$ maps of Figures 1a and $1 \mathrm{~b}$. The very close correspondence between the $\mathrm{mm}$ regions and the magnetic regions is quite clear. Detailed studies (Kundu, 1970) indicate that (a) the size of the $\mathrm{mm}$ regions is very closely related to the Ca-plage regions as well as the magnetic regions; (b) the excess brightness temperatures of the 3.5 and $9 \mathrm{~mm}$ regions is proportional to the magnetic field strength of the plage regions; and (c) the excess brightness temperatures at 3.5 and $9 \mathrm{~mm}$ are very poorly correlated with magnetic fields and areas of associated sunspots.

One of the most important characteristics of most $3.5 \mathrm{~mm}$ and some $9 \mathrm{~mm}$ maps is the existence of 'temperature depressions' or 'absorption features'. These features usually correspond to dark disk filaments observed in $\mathrm{H} \alpha$. As we know, the dark filaments form in regions of zero longitudinal magnetic field; the filamentary material is supported by a magnetic field parallel to the surface of the Sun. It is relevant to note that the temperature contours between the two components of a $m m-\lambda$ double source 


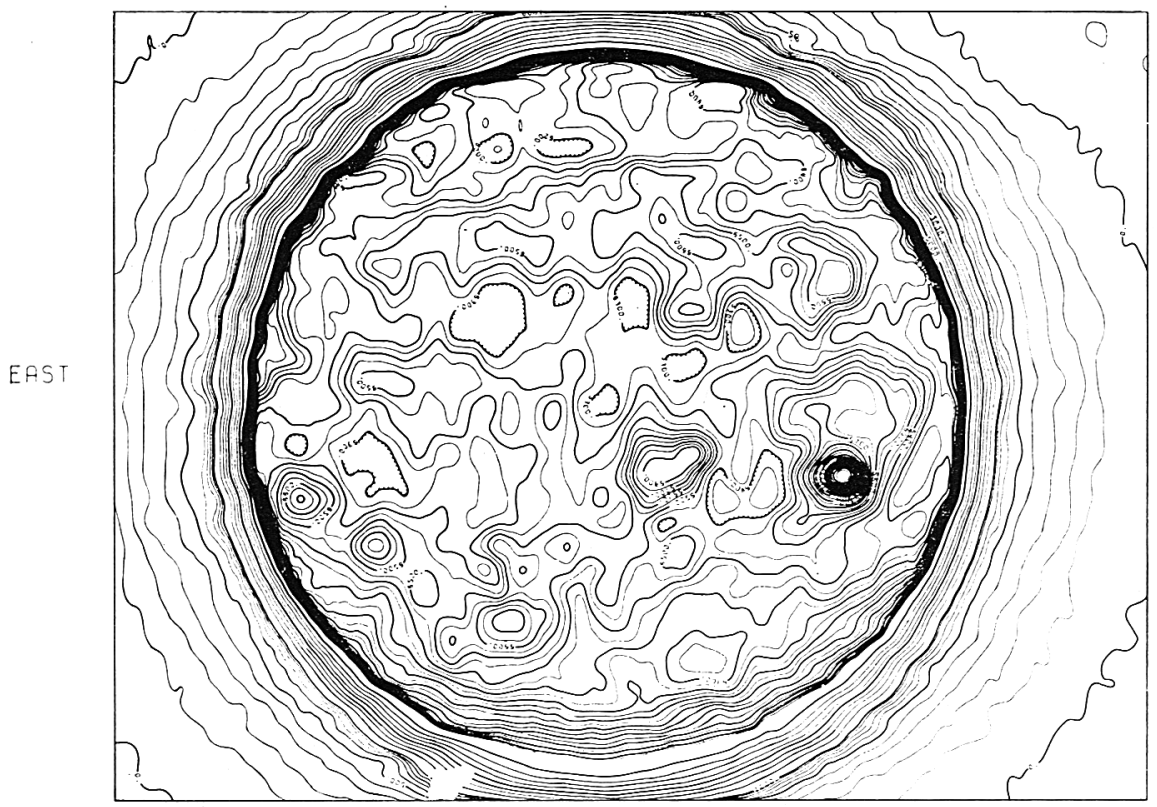

SOUTH

Fig. 1b. Solar Map at $3.5 \mathrm{~mm}-\lambda$ for Sept. 17, 1969. The grid used was $45^{\prime}$ by $37^{\prime}$ in RA and $\delta$ at intervals of $1^{\prime}$ arc in $\delta$. The regions marked by small dashes indicate temperature depressions (from Kundu, 1970).

very often delineates the boundary of the neutral region of the magnetic bipolar region even when the absorption feature is not so obvious. If we interpret the absorption as being caused by free-free absorption of the radio radiation by the filamentary material having high electron density and lower temperature, then we may have a method of estimating the electron density in the filament from the observed amount of absorption at $3.5 \mathrm{~mm}$ wavelength. The mean optical depth $\tau$ is given by $\tau=K l, K$ being the absorption coefficient and $l$ the dimension of the filamentary material through which the radio radiation passes. Since $K$ depends upon the wavelength $\lambda$, electron density $N_{e}$ and electron temperature $T_{e}$, therefore assuming an appropriate value of $T_{e}$, one can determine $N_{e}$. If one can measure the absorption at a second wavelength with comparable angular resolution, one can estimate both $N_{e}$ and $T_{e}$. The measurement of absorption at a number of millimeter wavelengths should lead to a fairly good estimate of the height of the disk filaments, since at a certain wavelength the absorption will cease to be observable.

The properties of the slowly varying radiation at millimeter wavelengths seem to be consistent with the thermal origin of this radiation in the higher than normal density regions at chromospheric temperatures. Indeed, the spectrum of brightness 

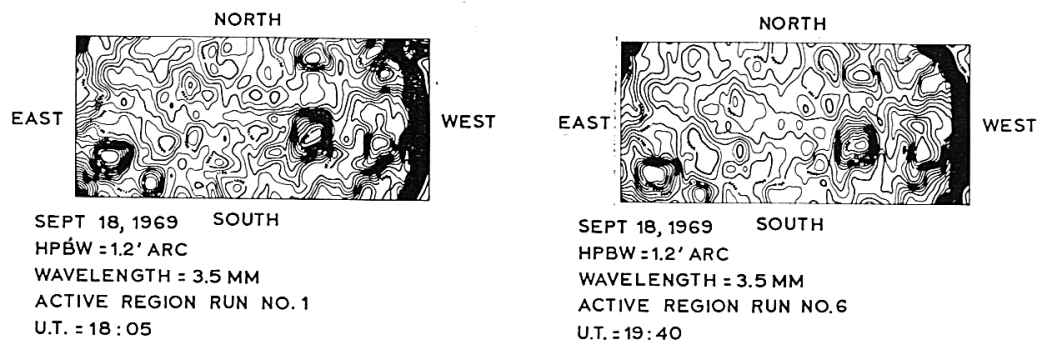

$$
\text { SEPT } 18,1969 \text { SOUTH }
$$

HPBW $=1,2^{\prime}$ ARC

WAVELENGTH $=3.5 \mathrm{MM}$

ACTIVE REGION RUN NO.6

U.T. $=19: 40$
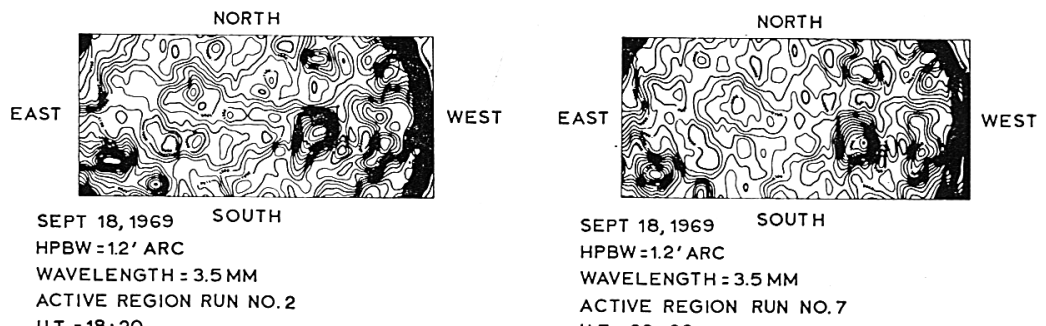

$$
\text { SEPT 18, } 1969 \text { SOUTH }
$$

HPBW $=1.2^{\prime}$ ARC

WAVELENGTH $=3.5 \mathrm{MM}$

ACTIVE REGION RUN NO.7

U.T. $=18: 20$

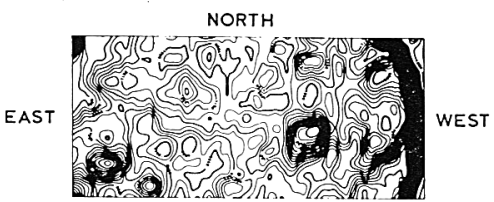

SEPT 18,1969 SOUTH

HPBW $=1.2$ ' ARC

WAVELENGTH $=3.5 \mathrm{MM}$

ACTIVE REGION RUN NO. 3

U.T. $=18: 40$
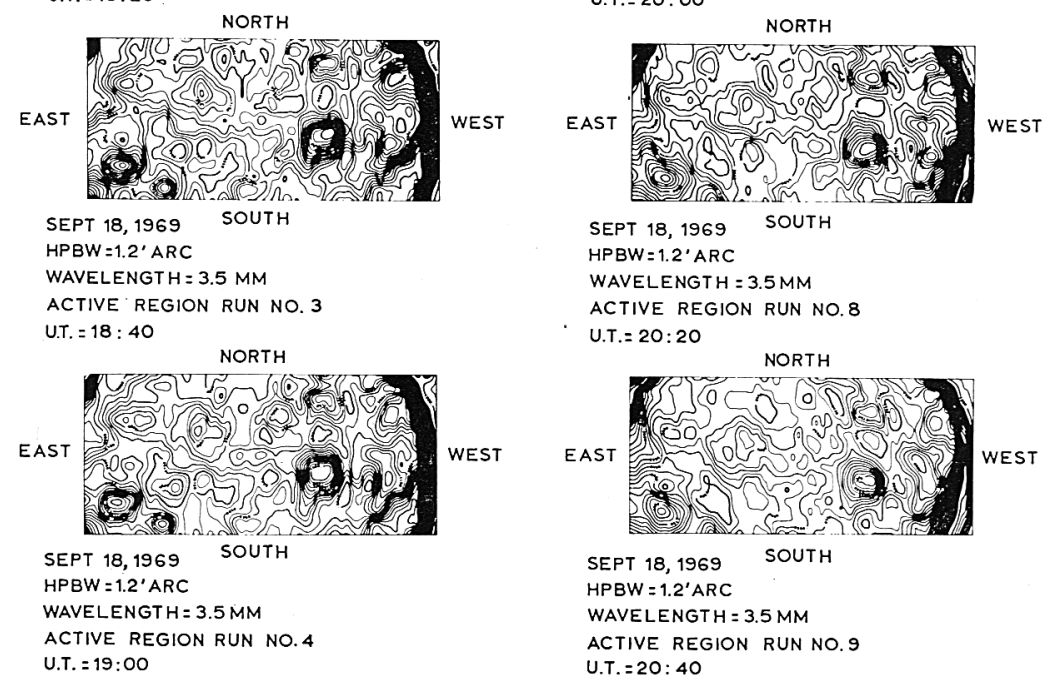

$$
\text { SEPT 18, } 1969 \text { SOUTH }
$$

HPBW $=1.2^{\prime}$ ARC

WAVELENGTH $=3.5 \mathrm{MM}$

ACTIVE REGION RUN NO.8

U.T. $=20: 20$

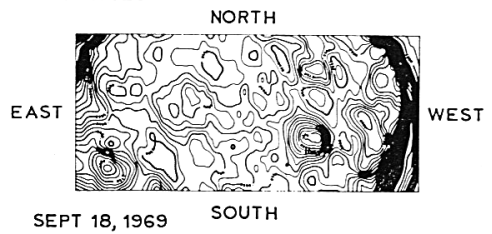

HPBW $=1.2$ 'ARC

WAVELENGTH $=3.5 \mathrm{MM}$

ACTIVE REGION RUN NO.9

U.T. $=20: 40$
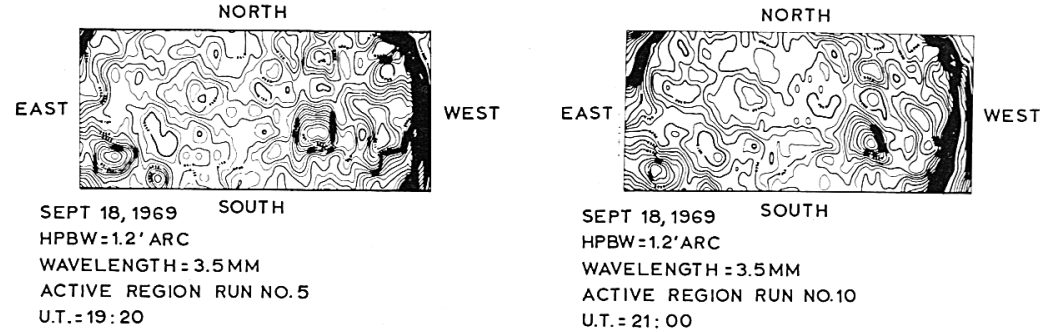

Fig. 2. A typical set of active region maps at $3.5 \mathrm{~mm}$ wavelength, illustrating the time evolution of active region. 


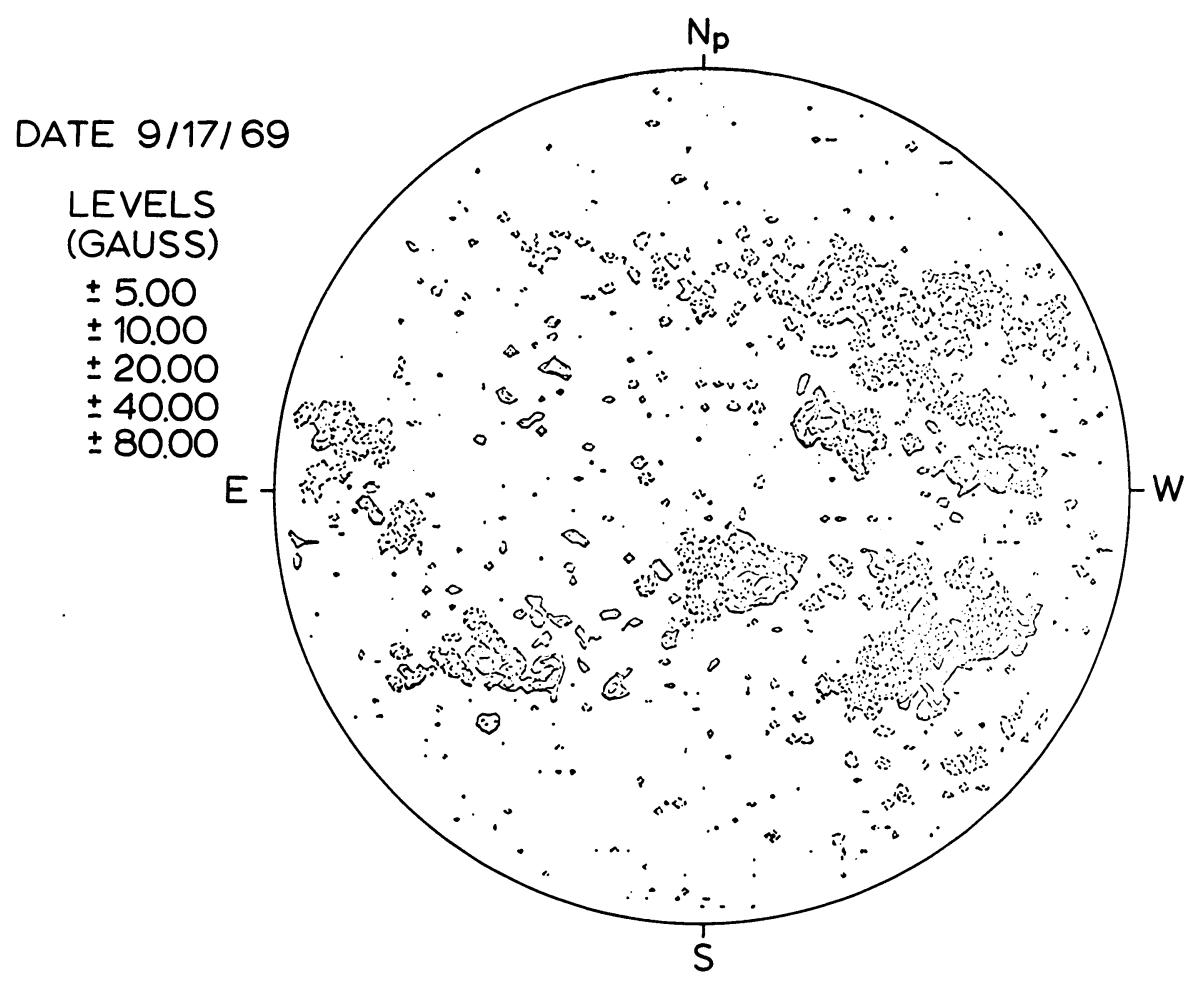

Fig. 3. Mount Wilson magnetogram for Sept. 17, 1969. The angular resolution used was 17" arc.

temperature which decreases with decreasing wavelength is compatible with this explanation. It is quite obvious that the gyroresonance absorption which is invoked to explain the slowly varying component at centimeter wavelengths cannot play any role at millimeter wavelengths, since the required magnetic field is at least an order of magnitude stronger than observed. This interpretation is strengthened by the fact that there is poor correlation between brightness temperature and sunspot magnetic field. The thermal radiation at millimeter wavelengths in the presence of a magnetic field should be circularly polarized; the degree of circular polarization can be used to measure the longitudinal fields in the chromosphere. Indeed for quasi-longitudinal propagation of radio waves in the magneto-ionic medium of the chromosphere, the degree of polarization is related by a simple expression to the magnetic field (see for example, Kundu, 1965). As we have noted earlier, the millimeter wavelength regions show very good correspondence with the regions of longitudinal magnetic fields on magnetograms; however, the angular resolution used for magnetograms is $17^{\prime \prime}$ as against 3.5 and 1.2 for 9 and $3.5 \mathrm{~mm}$ maps. In order to compare the two kinds of observations in a more meaningful manner, we have smoothed the magnetogram shown in Figure 3, with equivalent beams corresponding to those of 3.5 and $9 \mathrm{~mm}$ observations. The resulting 'smoothed' maps are shown in Figures 4 and 5. A compa- 


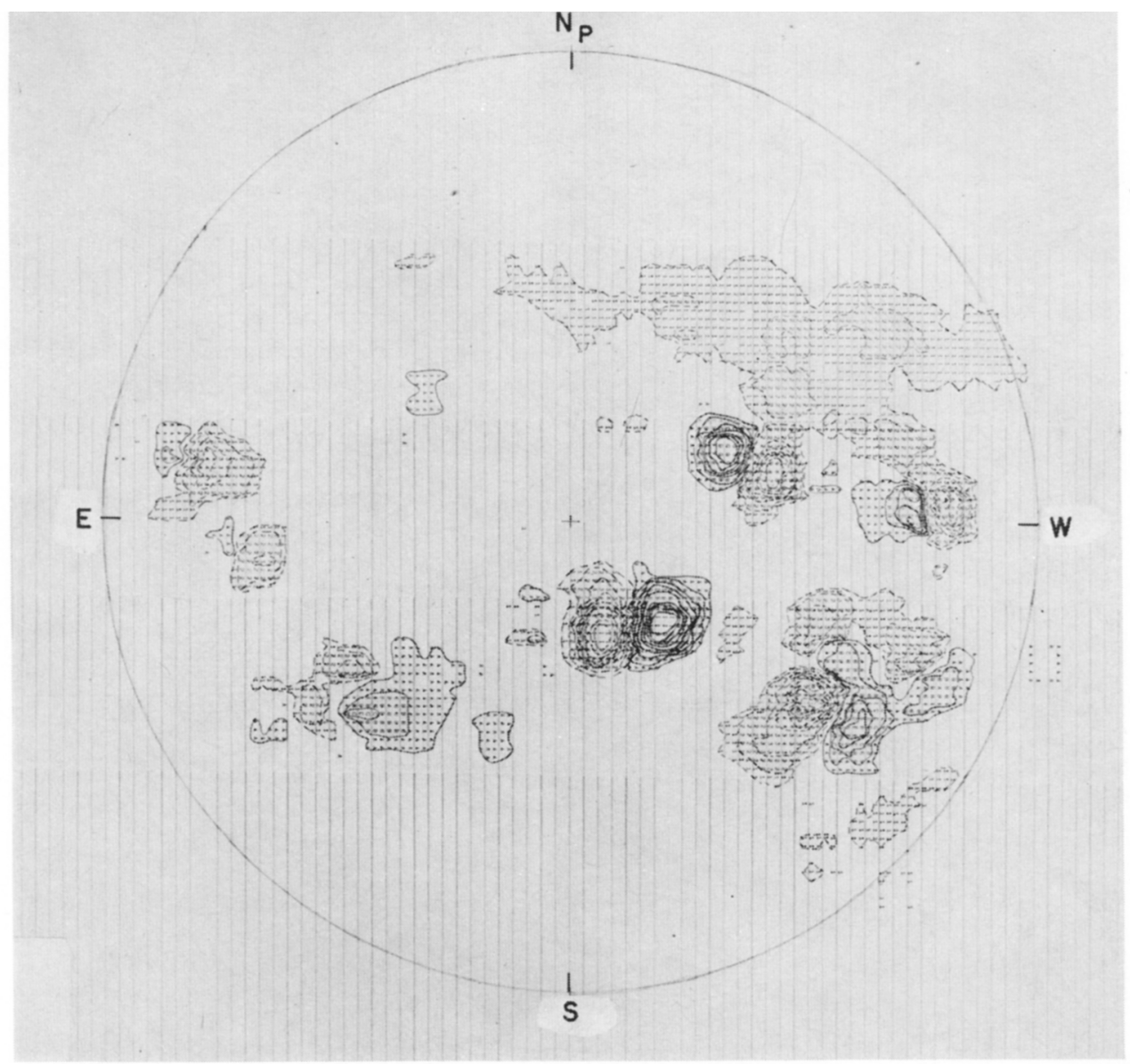

Fig. 4a. Magnetogram of Sept. 17, 1969 (shown in Figure 3), smoothed by a pencil beam of $1^{\prime} .2$ arc. The regions delineated by continuous and broken lines correspond to north and south polarities respectively. The outermost contour corresponds to a magnetic field of 0.03-3 G; the successive inner contours correspond to magnetic fields of 3-9, 9-15, 15-21, 21-27, 27-33, 33-39, 39-45 and $>45 \mathrm{G}$ respectively. 


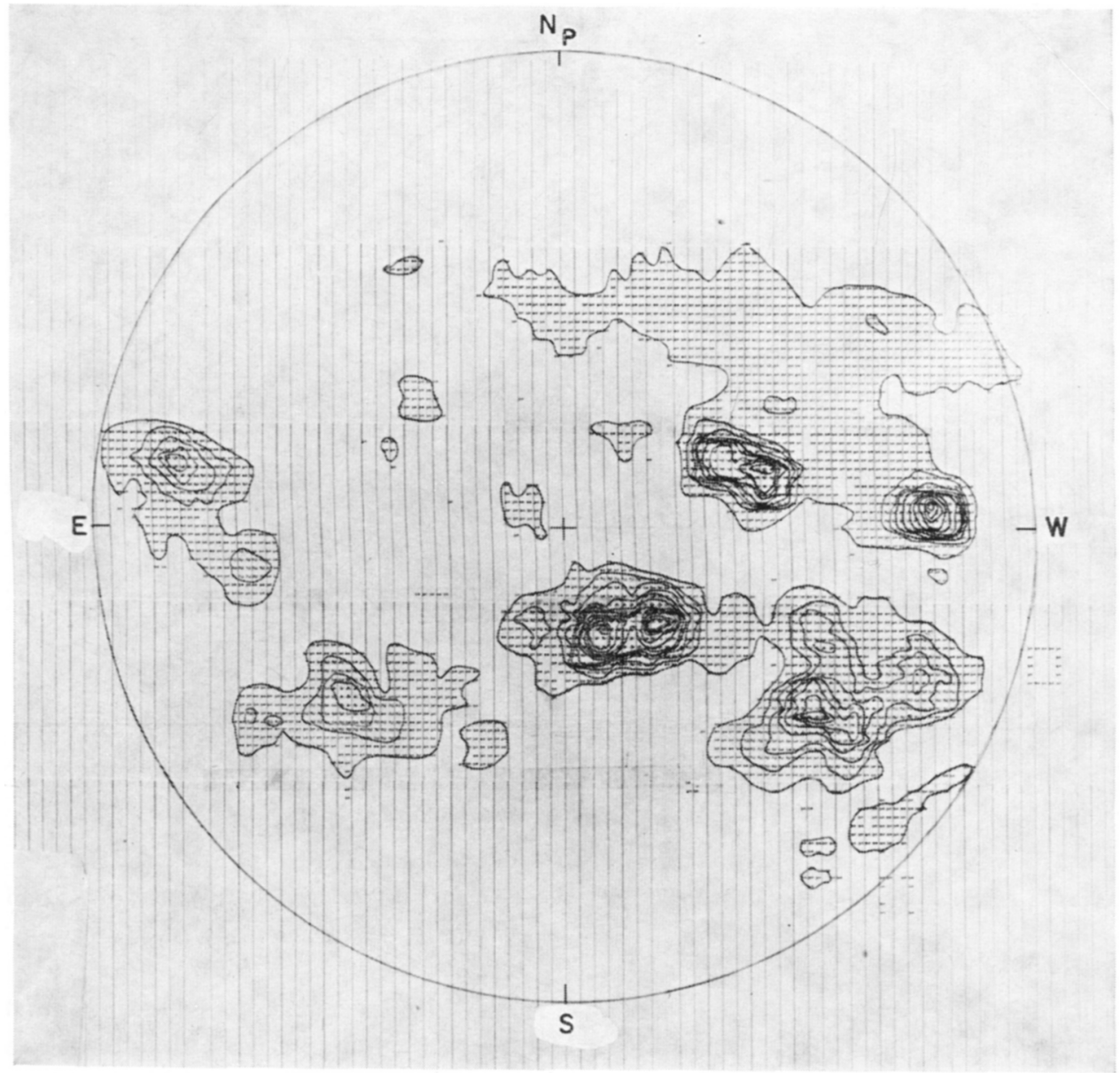

Fig. 4b. Same as Figure 4a, except that opposite polarities are not distinguished here, for direct comparison with the $3.5 \mathrm{~mm}$ map. The outermost contour corresponds to a magnetic field of $0.03-3 \mathrm{G}$; the successive inner contours correspond to magnetic fields of 3-9, 9-15, 15-21, 21-27, 27-33, 33-39. $39-45,45-51,51-57,57-63,63-69$, and $69-75$ G respectively. 


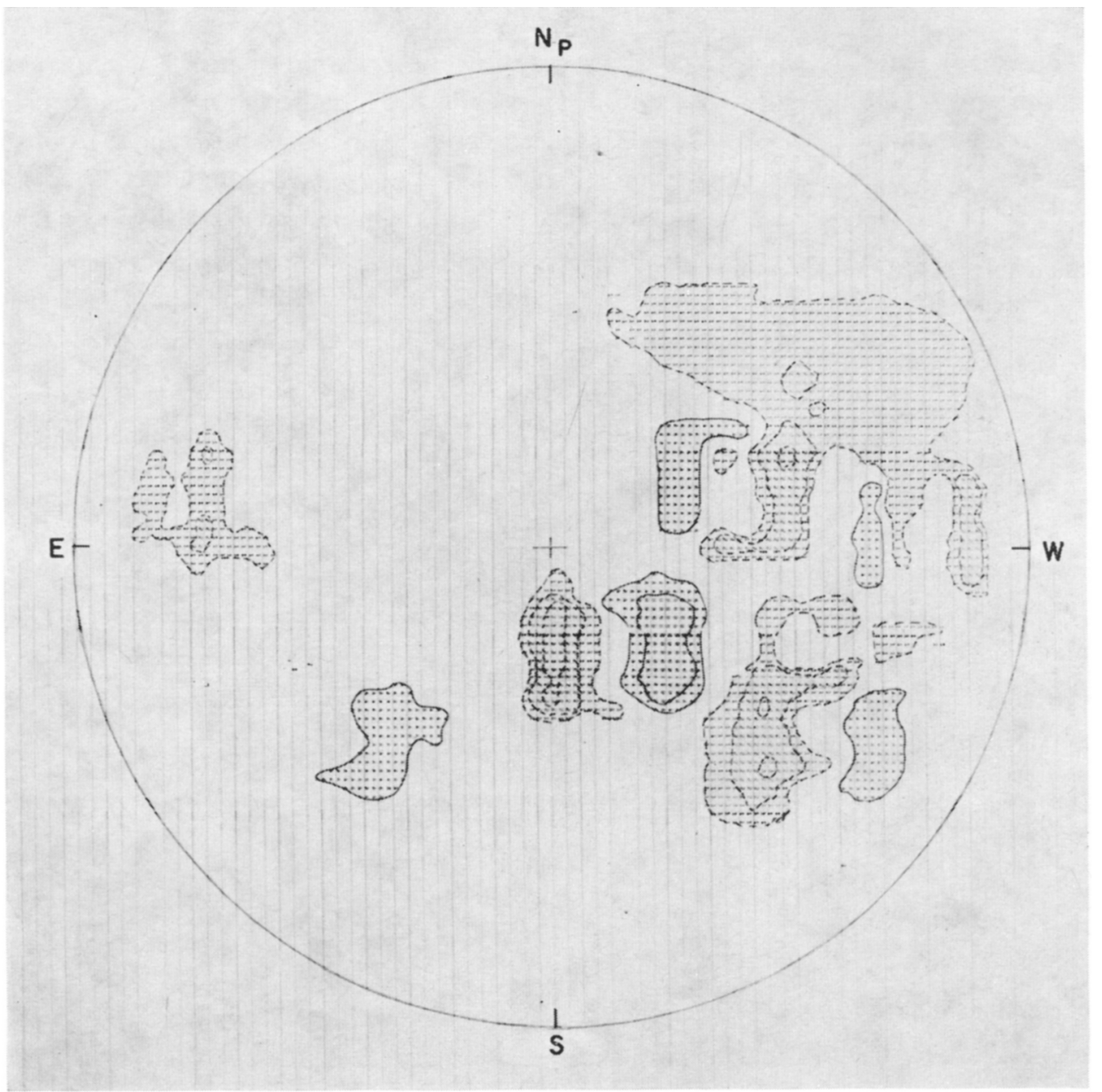

Fig. 5a. Magnetogram of Sept. 17, 1969 (shown in Figure 3), smoothed by a pencil beam of 3'.5 arc. The regions delineated by continuous and broken lines correspond to north and south polarities respectively. The outermost contour corresponds to $0.03-3 \mathrm{G}$; the successive inner contours correspond to 3-9, 9-15 and 15-21 G respectively. 


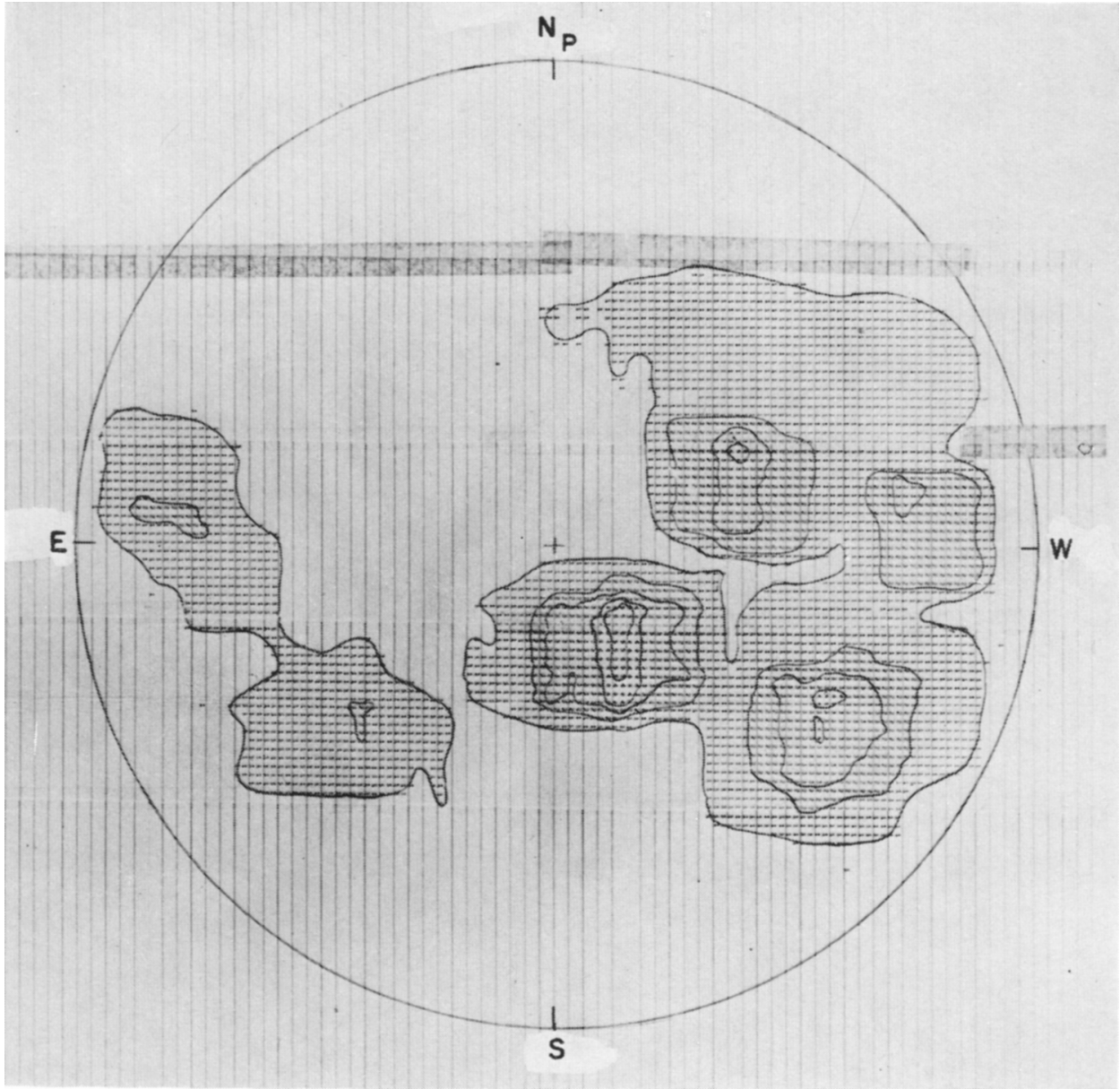

Fig. 5b. Same as Figure 5a except that opposite polarities are not distinguished here, for direct comparison with the $9 \mathrm{~mm}$ map. The outermost contour corresponds to 0.03-3 G; the successive inner contours correspond to $3-9,9-15,15-21,21-27,27-33$ and 33-39 G respectively. 
rison of these maps with those of Figure 1 establishes the one-to-one correspondence between the 3.5 and $9 \mathrm{~mm}$ active regions on the hand, and the magnetic regions on magnetograms on the other. Thus, the assumption of quasi-longitudinal propagation seems to be justified. Consequently, if one can measure the degree of circular polarization at 3.5 and $9 \mathrm{~mm}-\lambda$, one should be able to get fairly good estimates of longitudinal magnetic fields at corresponding heights in the chromosphere.

\section{References}

Kundu, M. R.: 1965, Solar Radio Astronomy, John Wiley-Interscience Publishers, p. 234.

Kundu, M. R.: 1970, Solar Phys. 13, 348. 